\title{
Kaposi's Disease (KS) in a Senegalese Child Living with HIV
}

\author{
F. Ly1 ${ }^{1}$, B. Niang², Y. Keita ${ }^{3}$, C. Dial ${ }^{4}$, A. Sow ${ }^{1}$, A. Sakho Kane', B. S. Ndiaye ${ }^{5}$, A. Sylla ${ }^{3}$, B. Camara ${ }^{1}$, \\ A. Ly Ba ${ }^{6}$, O. Ndiaye ${ }^{2}$
}

${ }^{1}$ Pediatric Service of the Pikine National Hospital, Dakar, Senegal

${ }^{2}$ Pediatric Department of the Albert Royer National Children's Hospital, Dakar, Senegal

${ }^{3}$ Pediatric Department of the Aristide Dantec Hospital Center, Dakar, Senegal

${ }^{4}$ Department of Anatomopathological of General Hospital Idrissa Pouye, Dakar, Senegal

${ }^{5}$ Department of Dermatology of the Pikine National Hospital Center, Dakar, Senegal

${ }^{6}$ Radiology Department of the Albert Royer National Children's Hospital, Dakar, Senegal

Email: *fatouly@ymail.com, drniangbacar@gmail.com, younousskeith@yahoo.fr, dialcherif@yahoo.fr, mimiesow024@gmail.com, adjaratoukane@yahoofr, sadikhndia ye@yahoo.fr, drassanesylla@gmail.com,

bccamara@yahoo.com, alyba2008@hotmail.fr, oussoubane@gmail.com

How to cite this paper: Ly, F., Niang, B., Keita, Y., Dial, C., Sow, A., Kane, A.S., Ndiaye, B.S., Sylla, A., Camara, B., Ly Ba, A. and Ndiaye, O. (2020) Kaposi's Disease (KS) in a Senegalese Child Living with HIV. Open Journal of Pediatrics, 10, 85-93. https://doi.org/10.4236/ojped.2020.101007

Received: November 25, 2019

Accepted: January 17, 2020

Published: January 20, 2020

Copyright $\odot 2020$ by author(s) and Scientific Research Publishing Inc. This work is licensed under the Creative Commons Attribution International License (CC BY 4.0).

http://creativecommons.org/licenses/by/4.0/ (c) (i) Open Access

\begin{abstract}
Kaposi's Disease or Kaposi's Sarcoma (SK ) is a multifocal malignant proliferation induced by viral growth factors, including interleukin 6 of human herpes virus type 8 (HHV8). We describe four forms of this disease who poses a real public health problem in East and Central Africa. The purpose of our observation was to report a rare condition in a Senegalese HIV-positive child. It was an 11-year-old girl from a region in central Senegal. She was an orphan of both parents, tested and monitored since the age of 5 for HIV infection 1. She was on the 1st line protocol. Due to a lack of support and good observance, she was referred to us at the age of 11 for follow-up in our structure in the suburbs of Dakar. The initial follow-up assessment showed a very low CD4 count and a very high viral load. Before the lack of clinical and immune-virological response, a genotypic resistance test was performed and showed immunological and virological failure. The initial development was marked by the appearance of lesions which were highly suggestive of Kaposi's disease. She was on 2nd line treatment. The histopathological aspect of cutaneous biopsy was very suggestive of Kaposi's disease. The subsequent course after ART and bleomycin treatment was clinically marked by regression of skin lesions. Virologically, it was marked by a fall in the viral load. Immunologically there was a gradual recovery of CD4 levels which came back to normal. Our observation demonstrates that absence of effective antiretroviral therapy for HIV increases the risk to develop Kaposi's sarcoma.
\end{abstract}


Keywords

Kaposi's Sarcoma, Antiretroviral Therapy, HIV, Child

\section{Introduction}

Kaposi's Disease or Kaposi's Sarcoma (SK ) is a multifocal malignant proliferation induced by viral growth factors, including interleukin 6 of human herpes virus type 8 (HHV8) [1]. We describe four forms of this disease: a classical Mediterranean form discovered by the Hungarian dermatologist Moricz Kaposi, who for the first time in Vienna, Austria, described in 1872 a rare malignant skin disease of a particular type which affects elderly men and is present in the form of macules or nodules of purplish red or brown appearance; an endemic form described in 1950 in central and eastern Africa, around the Great Lakes affecting young adult men and children. That is called a post-transplant form related to iatrogenic acquired immunosuppression, described in 1970 in transplant patients treated with immunosuppressive drugs and an AIDS-related epidemic form, described in 1981 in HIV-infected persons [2]. It is one of the most frequent opportunistic diseases and the first cancer in HIV-infected patients and the first cutaneous cancer in sub-Saharan Africa before cutaneous carcinomas and melanoma [3]. Kaposi's Disease poses a real public health problem in East and Central Africa where in some regions it accounts for $20 \%$ to $50 \%$ of all diagnosed cancers [4]. Few studies have been conducted on Kaposi's disease in children infected with HIV in Africa. In Mali in 2007, a study mentioned a prevalence of $1.6 \%$ [5].

The purpose of our observation was to report a rare condition in a Senegalese HIV-positive child.

We were able to obtain informed consent from the respondent who was the maternal aunt.

\section{Observation}

It was an 11-year-old girl from a region in central Senegal who attended Koranic school. She was the 2 nd of 2 siblings when the elder child was alive and well. She was an orphan of both parents, and was tested and monitored since the age of 5 for HIV infection 1. She was on the 1st line protocol: Zidovidine (AZT)-Lamivudine (3TC)-Efavirenz (EFV). Due to a lack of support and good observance, she was referred to us at the age of 11 for follow-up in our structure in the suburbs of Dakar. On admission, she had a weight loss of $23 \mathrm{~kg}$ for a height of $133.5 \mathrm{~cm}$ and multiple dental decays. The initial follow-up assessment showed a very low CD4 count of $159 / \mathrm{mm}^{3}$ and a very high viral load of 94.310 copies/ml. His treatment was adjusted according to the regimens: AZT-3TC: form $300 / 1501 \mathrm{cpr}^{1 / 2}$ day and $1 / 2$ EFV $600 \mathrm{mg}$ form cpr daily in the evening and cotrimoxazole $1 \mathrm{cpr}$ day. Before the lack of clinical and immune-virological re- 
sponse, a genotypic resistance test was performed after 6 years and showed immunological and virological failure with a strain resistant to nucleoside reverse transcriptase inhibitors (NRTIs) and non-nucleoside reverse transcriptase inhibitors (NNRTIs) (Figure 1).

The initial development was marked after 15 months by the appearance of oedemas sitting in the upper limbs predominating at the wrists and at lower extremities that are more marked at the ankles, which become hard if not taking the scab with pain in limb mobilization without functional impotence. After 8 months, hyperpigmented plaques and erythematous-angiomatous nodules appeared (Figure 2(a), Figure 2(b)).

There was no involvement of the mucous membranes, particularly the mouth and conjunctiva. These lesions were highly suggestive of Kaposi's disease. She was on 2nd line treatment according to the Didanosine (DDI)-Abacavir (ABC)

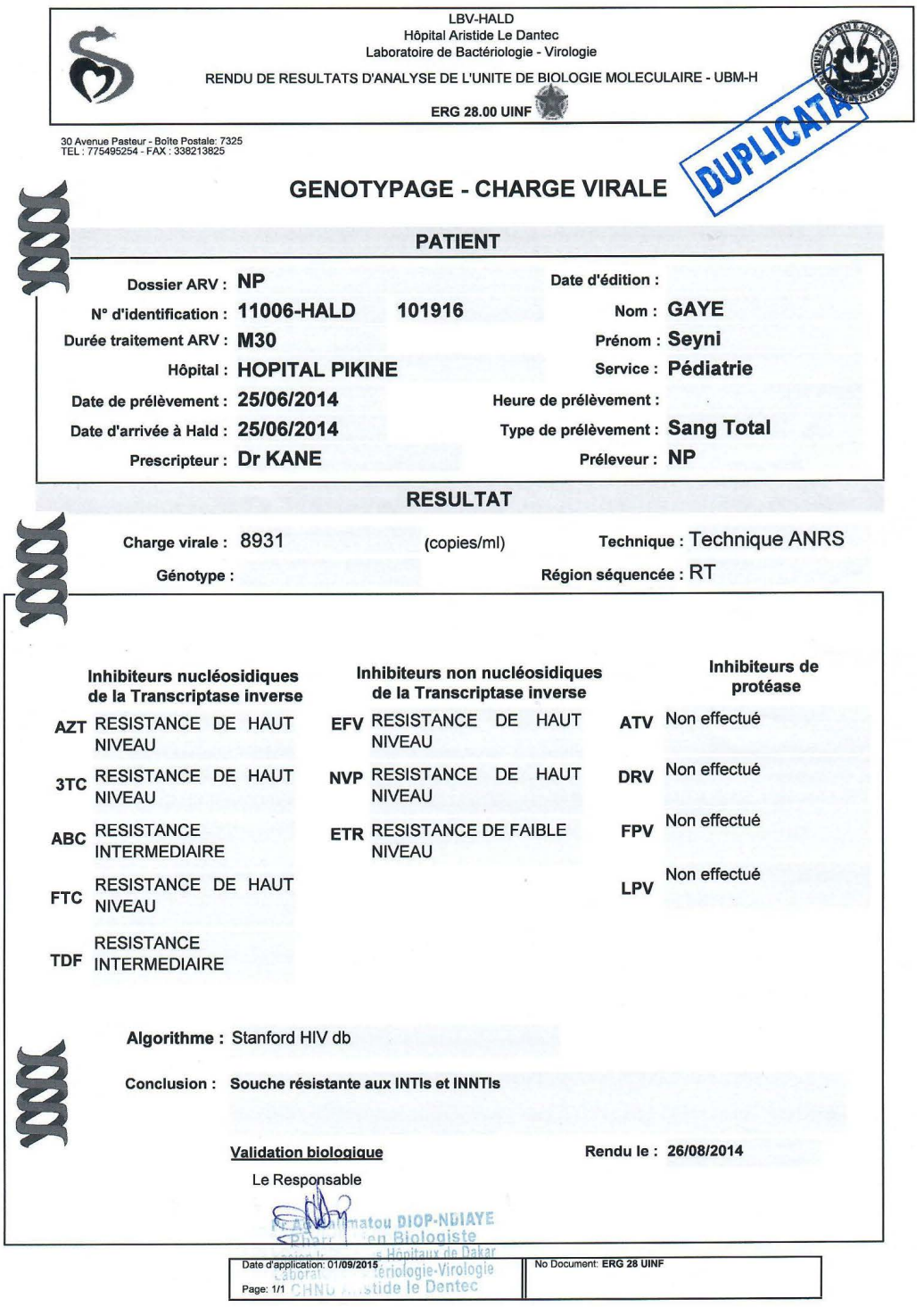

Figure 1. Genotypic resistance test. 

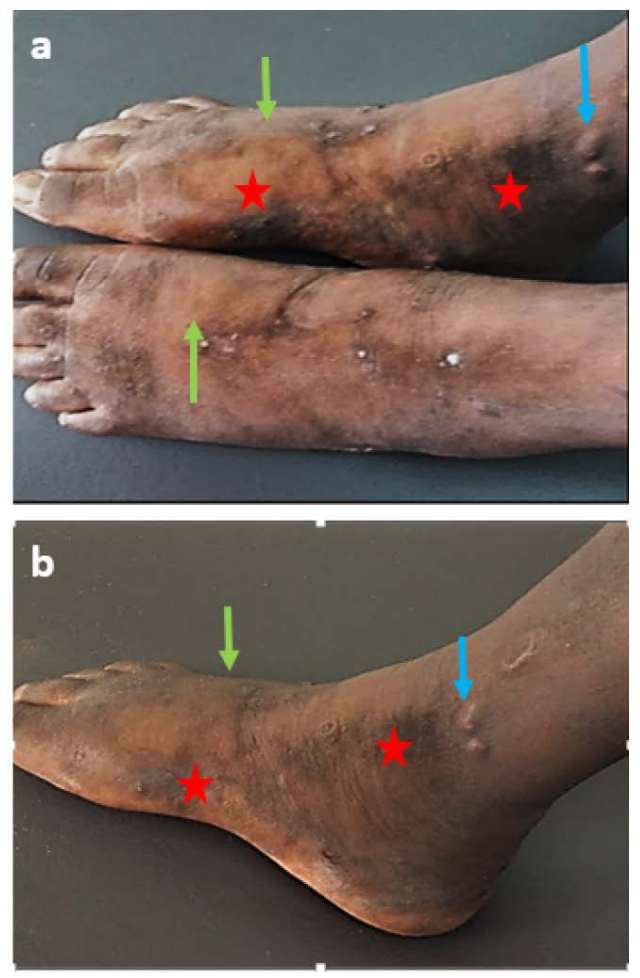

Figure 2. (a) (b): edemas $(\rightarrow)$ of the lower limbs, hyperpigmented plaques $(\star)$ and erythematous-angiomatous nodnules $(\longrightarrow$ ).

Lopinavir/Ritonavir (LPV/r) regimen. Cutaneous biopsy performed 4 months after the introduction of the new protocol showed an unencapsulated intradermal tumor nodule consisting of fibroblastic cells and especially blood microvessels with blood extravasation and hemosiderin deposits by points. The histopathological aspect was very suggestive of Kaposi's disease (Figure 3). She had received bleomycin treatment at $10 \mathrm{IU}$ per dose every 15 days in MI in 8 courses.

The subsequent course after cures and after 5 months of ART was clinically marked by regular weight gain and regression of skin lesions. Virologically, it was marked by a fall in the viral load less than 50 copies per $\mathrm{ml}$, which had become undetectable after 8 months. Immunologically there was a gradual recovery of CD4 levels which came back to normal. Currently after 4 years of follow-up she is still in clinical and biological remission under the same antiretroviral protocol.

\section{Discussion}

In terms of epidemiology, co-infection with Acquired Immunodeficiency Syndrome (AIDS) and Kaposi's sarcoma is not uncommon in Europe, but is rare in Africa. It was reported in Africa by Bouquety et al in Central Africa, Marquart et al. in Uganda and Dicko et al. in Mali among young children infected with HIV, aged 11 months, 2 years, 1 month and 5 years respectively [6] [7] [8]. A predominance of the young population was also observed in adults by most authors 


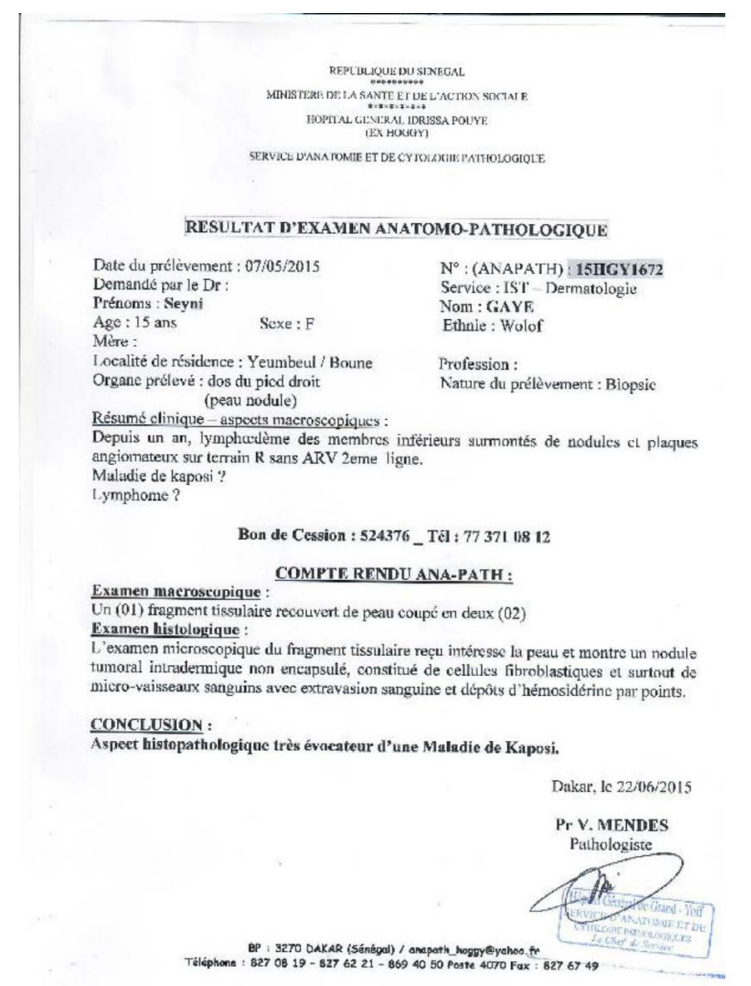

(a)

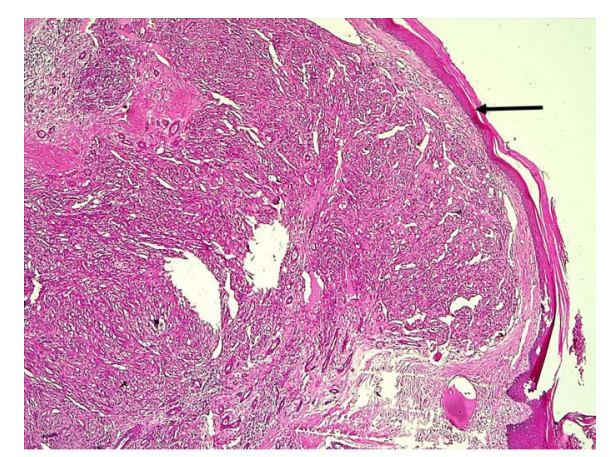

(b)

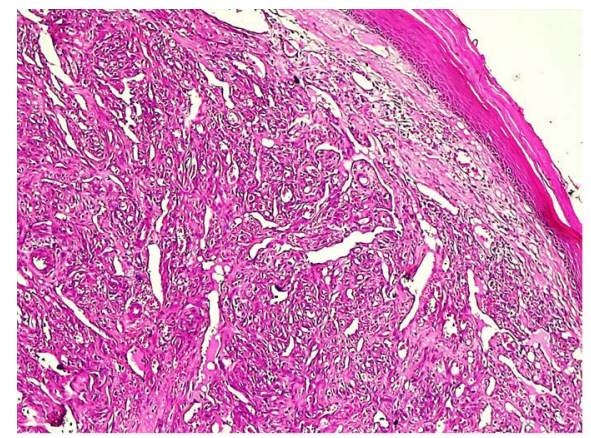

(c)

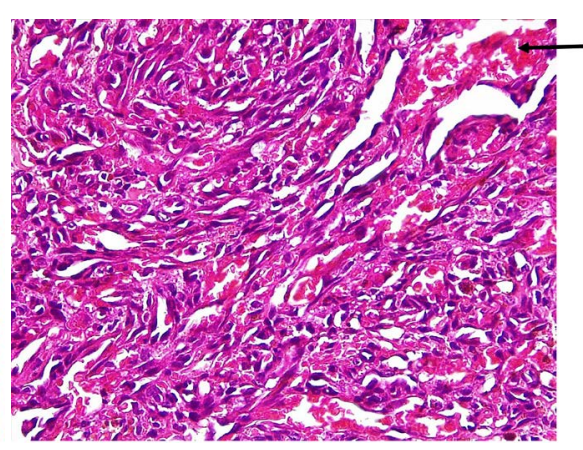

(d)

Figure 3. (b) Kaposi cutané: prolifération de vaisseaux capillaires et de cellules fusiformes. HE $\times 100$; (c) Kaposi cutané: prolifération de vaisseaux capillaires et de cellules fusiformes sous l'épiderme (fleche). HE $\times 150$; (d) Kaposi cutané: prolifération de vaisseaux capillaires et de cellules fusiformes avec foyers d'extravasation sanguine (fleche). HE $\times 250$. 
with mean ages ranging from 33.5 to 38.5 years [9] [10]. This high incidence of juvenile involvement could be explained by the fact that youth is a sexually more active population and therefore more exposed to sexually transmitted infections, unlike our patient, whose first signs of the disease had appeared at an age when she was not sexually active. Vertical transmission appeared to be the most likely mode of contamination.

Clinically, cutaneous involvement is inaugural in $90 \%$ of cases. The elementary lesions are of type: brown or purple brown macules on pigmented skin, pigmented papulo-nodules, angiomatous nodules, infiltrated or verrucous placard or ulcero-papular. All these lesions can reach all the integuments especially the trunk, the limbs (extremities: hands, feet), and the face (nose and eyelids). Lymphedema of the affected limb may be revealing. The mucosal involvement is also frequently observed, in $50 \%$ of the cases with type of bucco-pharyngeal (palatal, gingival, labial, jugal, lingual, tonsillar) involvement, which can ulcerate and to be superinfected, of genital involvement and ocular involvement [11]. The isolated cutaneous localization was observed in our patient in the form of hyperpigmented closets, erythematoangiomatous nodules and lymphedema. Dicko et al. in Mali also reported a more extensive Kaposi skin disease revealing HIV in a child with a polymorphic rash of papules and nodules on the face, trunk, back and limbs [8] These same nodular lesions followed by purplish macules were reported by Karabinta et al. [9]. Tounouga et al. in Cameroon reported lesions mainly localized to the skin, and more rarely to the mucous membranes. The main clinical features were lymphoedema (35\%), papulo-nodules $(25.8 \%)$ and erythematous-angiomatous macules (14.3\%) [12]. These findings differ from those of the literature, which state that the most common elemental lesion is a macula that progresses to nodules and placards [5]. In Mali, a recent study reported a predominance of cutaneous localization followed by mucocutaneous and mucocutaneous forms (with predominance of oral location) [9]. On the other hand, an older study had found a predominance of cutaneo-mucous localization [5]. Ganglionic involvement and visceral involvement are frequent, especially digestive and pulmonary. Gastrointestinal involvement is noted in 50\% of cases of Kaposi diseases with cutaneous involvement and in $100 \%$ of cases with oral location.

It can be asymptomatic or revealed by pain, diarrhea, digestive haemorrhage, with endoscopic examination of papules or red angiomatous nodules, sitting in the stomach, duodenum, colon, rectum [11]. In Africa, Marquart et al. in Uganda had observed a lymphadenopathic form of Kaposi's sarcoma without skin lesions in a young HIV-positive Ugandan boy with severe infection of the respiratory tract, lymph nodes and spleen [7]. Boukety et al. in Central Africa had also reported Kaposi lymphocutaneous sarcoma marked by chronic diarrhea and disseminated lymphadenopathy, gingivostomatitis with herpes vesicles, hepatosplenomegaly, and cervico-axillo-inguinal lymphadenopathy and cutaneous nodules in dark brown or black velvet [6]. The pulmonary involvement occurs in $20 \%$ to $50 \%$ of cases, revealed by a dry cough, dyspnea, acute respiratory distress 
syndrome suggestive of pneumocystosis, with non-specific radiological signs such as bilateral interstitial or alveolar infiltrate and nodular images poorly limited. All organs can be affected, in particular otorhinolaryngological, medullary, urogenital and bone [11]. Karabinta et al. had observed multifocal localization in $76.4 \%$ of cases, especially in HIV-positive patients [9]. This result proves that MK is multifocal neoplasia [1].

Immunologically, only the low CD4 count was selected as having a significant impact on the occurrence of Kaposi's Disease [11]. Dicko et al. also reported that our patient had a low CD4 count $\left(240 / \mathrm{mm}^{3}\right)$ in an HIV-infected young boy with Kaposi [8]. On the other hand, a CD4 cell count $\geq 500$ cells $/ \mathrm{mm}^{3}$ represents a lower risk of developing the disease [11]. Histologically, the diagnosis of Kaposi's Disease is based on evidence of a proliferation of irregularly anastomotic vascular slits, between collagenous masses and around normal dermal vessels and appendages, mixed together with fusiform cell bundles, a predominantly lymphocytic, mononuclear inflammatory infiltrate with extravasated red blood cells that, once phagocytosed, take the appearance of hyaline globules [11]. These lesions are identical to those found in our patient. Other authors have described these same lesions [6] [7].

Etiologically, the presence of HHV8 in tumor lesions of all forms of Kaposi's disease was demonstrated hence its name of herpes virus associated with Kaposi's sarcoma (KSHV). There was also evidence that HHV8 infection preceded the onset of Kaposi's disease, which has a developmental delay of approximately 5 to 6 years in HIV-positive patients. In addition, the prevalence level of anti HHV8 antibodies and the incidence of Kaposi's Disease are consistent. These arguments associated with molecular data have allowed HHV8 to be implicated as a causal agent of Kaposi's Disease.

HHV8 is an oncogenic virus belonging to the family Herpesviridae and the genus Rhadinovirus of which it is the only representative currently known in humans. This DNA virus encodes a whole host of proteins homologous to cellular oncogenes, which reinforces the hypothesis of its involvement in the appearance of cancerous lesions [13].

The modes of transmission by the HHV-8 virus are not yet fully understood. The possibility of transmission by insect bite is controversial. Transmission through saliva is possible because it contains large amounts of viral particles unlike other fluids (blood and genital secretions) regardless of CD4 count [13].

In young children, as in our patient, vertical transmission from mother to child during delivery and through the blood was more incriminated.

The virological evidence of HHV8 infection was not provided in our patient but it is based on serology (immunofluorescence, ELISA), the detection of viral proteins in target tissues, and genomic sequences by PCR [11].

Therapeutically, the management of AIDS-related Kaposi Disease is primarily based on the restoration of immunity by antiretroviral therapy, which has significantly modified the prognosis of the disease. The specific treatment of Kaposi's Disease is based on local means: surgery on limited lesions, few al- 
lowing histological examination; cryotherapy with liquid nitrogen; cryosurgery with nitrous oxide; fractional-dose radiotherapy and local chemotherapy with vinblastine. The most commonly used general means are bleomycinmono therapy; vincristine, epirubicin, etoposide, especially liposomal anthracyclines and taxones, which now tend to replace ABV-type chemotherapy (adriamycin + bleomycin + vincristine) [11].

On the evolutionary level, our patient had spectacularly evolved with ART and monotherapy. However, paradoxical aggravations of Kaposi's disease may be noted in the first months after initiation of antiretroviral therapy [11]. Bouquety et al reported a worsening of the clinical picture with dissemination of cutaneous nodules followed by pleuropneumopathy [6]. Kaposi Disease is also one of the most commonly reported opportunistic infections in immune restoration syndromes [11].

\section{Conclusion}

Kaposi's sarcoma is one of the most common opportunistic diseases and the first cancer in HIV-infected patients. Our observation demonstrates the importance of adherence to antiretroviral therapy in HIV-positive patients. In the absence of effective antiretroviral therapy for HIV, the risk of developing Kaposi's sarcoma is higher compared to the HIV-negative population.

\section{Conflicts of Interest}

The authors declare no conflicts of interest regarding the publication of this paper.

\section{References}

[1] Morand, J.J., Lightburn, E., Simon, F. and Potte, J.H. (2007) News about Kaposi Disease. Medecine Tropicale, 67, 123-130.

[2] Camparo, P., Gessain, A. and Arborio, M. (1996) Kaposi Sarcoma and Human Herpes Virus Type 8: Recent Acquisitions. Medecine Tropicale, 56, 231-237.

[3] Engels, E.A., Pfeiffer, R.M., Goedert, J.J., et al. (2006) Trends in Cancer Risk among People with AIDS in the United States 1980-2002. AIDS, 20, 1645-1654.

https://doi.org/10.1097/01.aids.0000238411.75324.59

[4] Pantanowitz, L. and Dezube, B.J. (2004) Advances in the Pathobiology and Treatment of Kaposi Sarcoma. Current Opinion in Oncology, 16, 443-449. https://doi.org/10.1097/00001622-200409000-00006

[5] Cissé, H., Dao, S., Oumar, A.A., et al. (2007) Kaposi's Disease at the Heart of HIV/AIDS in a Hospital in Bamako. Mali Medical, 22, 29-32.

[6] Bouquety, J.C., Siopathis, M.R., Ravisse, P.R., Lagarde, N., Georges-Courbot, M.C. and Georges, A.J. (1989) Lympho-Cutaneous Kaposi's Sarcoma in an African Pediatric AIDS Case. The American Journal of Tropical Medicine and Hygiene, 40, 323-325. https://doi.org/10.4269/ajtmh.1989.40.323

[7] Marquart, K.H., Müller, H.A., Hartter, P., Oku, J.C., Ayuko, W.O. (1987) Lymphadenopathic Type of Kaposi's Sarcoma in a Ugandan Child Seropositive for LAV/HTLV-III Antibodies. Journal of Tropical Medicine and Hygiene, 90, 93-94. 
[8] Dicko, A., Fofana, Y., Traoré, A., Berthé, S., Touré, S., Lamissa, C., Guindo, B., Keita, A. and Faye, O. (2017) Kaposi's Disease in an HIV Positive Child, with Probable Contamination from His Grandmother. Bulletin de la Société de pathologie exotique, 110, 247-249. https://doi.org/10.1007/s13149-017-0566-x

[9] Karabinta, Y., Faye, O., Traoré, Z., Touré, S., Dicko, A., Konaté, I., Dao, S. and Konare, H.D. (2018) Disease of Kaposi in Consultation of Dermatology in the University Hospital Gabriel Touré: Study of 36 Cases. Revue Malienne d'infectiologie et de microbiologie, 11, 57-59

[10] Asuquo, M.E. and Ebughé, G. (2009) Cutaneous Cancers in Calabar, Southern Nigeria. Dermatology Online Journal, 15, 11.

[11] Aubry, P. and Gaüzère, B.A. (2019) Kaposi Disease News 2019 Diploma in Tropical Medicine from Indian Ocean Countries. 1-5.

[12] Tounouga, D.N., Kouotou, E.A., Nansseu, J.R. and Bissek, A.C. (2018) Caractéristiques cliniques et déterminants de survenue de la maladie de kaposi au cours de l'infection par le VIH: Etude cas-témoin rétrospective de 16 ans à Yaoundé, Cameroun. Annales de Dermatologie et de Vénéréologie, 145, A44-A45.

[13] Jary, A., Dupin, N., Calcez, V. and Marcellin, A.G. (2019) Human Herpes Virus 8: Virological, Epidemiological, Clinical and Therapeutic Aspects. EMC-Infectious Diseases, 16, 1-12. 